\title{
Perioperative Approach in a Patient with Myasthenia Gravis
}

\author{
Saimir Kuci ${ }^{1 *}$ (iD), Alfred Ibrahimi ${ }^{1}$, Shaban Memeti ${ }^{2}$, Marsela Goga ${ }^{1}$, Selman Dumani ${ }^{3}$, Ali Refatllari ${ }^{3}$ \\ ${ }^{1}$ Department of Cardiac Anesthesia, Mother Teresa University Hospital Center, Tirana, Albania; ${ }^{2}$ University Clinic of Paediatric \\ Surgery, Faculty of Medicine, Ss Cyril and Methodius University of Skopje, Skopje, Republic of Macedonia; ${ }^{3}$ Department of \\ Cardiac Surgery, Mother Teresa University Hospital Center, Tirana, Albania
}

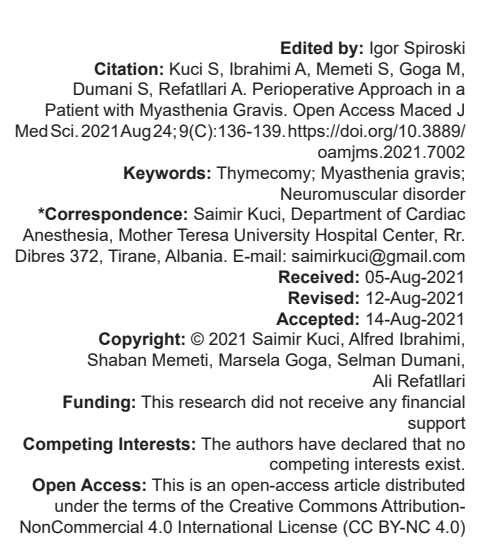

Introduction

The incidence of myasthenia gravis (MG) is cited as 50-142 cases per 1 million or 0.25 to 2.0 per 100,000 population [1], [2]. Gender discrepancy is present, and women 20-30 years of age are most often affected, followed by men who are often older than 60 years of age when their disease presents [1]. The disease is a chronic autoimmune disorder characterized by a decrease in acetylcholine receptors at the neuromuscular junction secondary to their destruction or inactivation by circulating antibodies [1]. Muscular weakness, fatigability, and rapid exhaustion especially after repetitive voluntary muscle use, as with exercise, are the hallmark signs of the disorder. Four Stages of the disease have been classified based on the severity of muscular involvement and range from self-limiting extraocular muscle involvement Stage I to severe whole-body generalized muscular weakness Stage IV [3]. Patients are at an increased risk of pulmonary aspiration because of pharyngeal and laryngeal muscle weakness, and they may present with dysphagia, dysarthria, and an overall difficulty of handling secretions. Cardiovascular involvement usually occurs and manifests in atrial fibrillation, heart block, and cardiomyopathy, with the presented case having hypertension as the only known cardiovascular manifestation.

Myasthenia gravis has historically been a challenging disease for the anesthetist to manage because of its involvement in the pulmonary, muscular, and cardiovascular systems as well as with drug interactions.

\section{Case Report}

An $\quad 87-\mathrm{kg}, \quad 168-\mathrm{cm}, \quad 38-y e a r-o l d$ woman presented with the main complaint of weakness of the entire limbs nausea, and vomiting then blurred vision for 7-8 months, symptoms that have worsened recently. On physical examination, there was right ptosis. Muscle strength based on the MRC scale was 3. After Neurologist consultation and tests that were made including rapid nerve stimulation examination, which showed a decremental decrease of $>10 \%$ in the abductor muscles of the minimi digit, trapezius, and right ocular orbicularis the patient was diagnosed with Myasthenia Gravis.

Chest CT scan was performed showing a visible heterogeneous mass with contrast enhancement 
in the anterior mediastinum with dimensions of $6 \times 4 \mathrm{~cm}$ in the thymus area suggestive of a thymoma.

Based on the modified Osserman classification, the patient was included in stage III. She received therapy with acetylcholinesterase inhibitors (pyridostigmine) with no clinical improvement and returned to the hospital complaining also of shortness of breath in efforts. In such conditions, she was consulted by the Neurologist and Cardiac surgeon to perform Thymectomia.

The patient underwent 3 sessions of Plasmapheresis, received Pyridostigmine and Corticosteroids therapy before surgery.

Preoperatively all examinations, blood cell count, chemistry laboratory values, and radiological examinations were normal.

Anesthesia was induced using fentanyl and propofol. Under general anesthesia, tracheal intubation was performed using topical analgesia without neuromuscular blocking drugs. Intraoperative monitoring included, routine ECG, arterial and central venous pressure, pulse oximetry, end-tidal carbon dioxide, and temperature. Anesthesia was maintained with sevoflurane and propofol. The patient was extubated $2 \mathrm{~h}$ later in the cardiosurgical intensive care unit.

Thymectomy was performed under a total surgical time of $35 \mathrm{~min}$ (Figure 1).

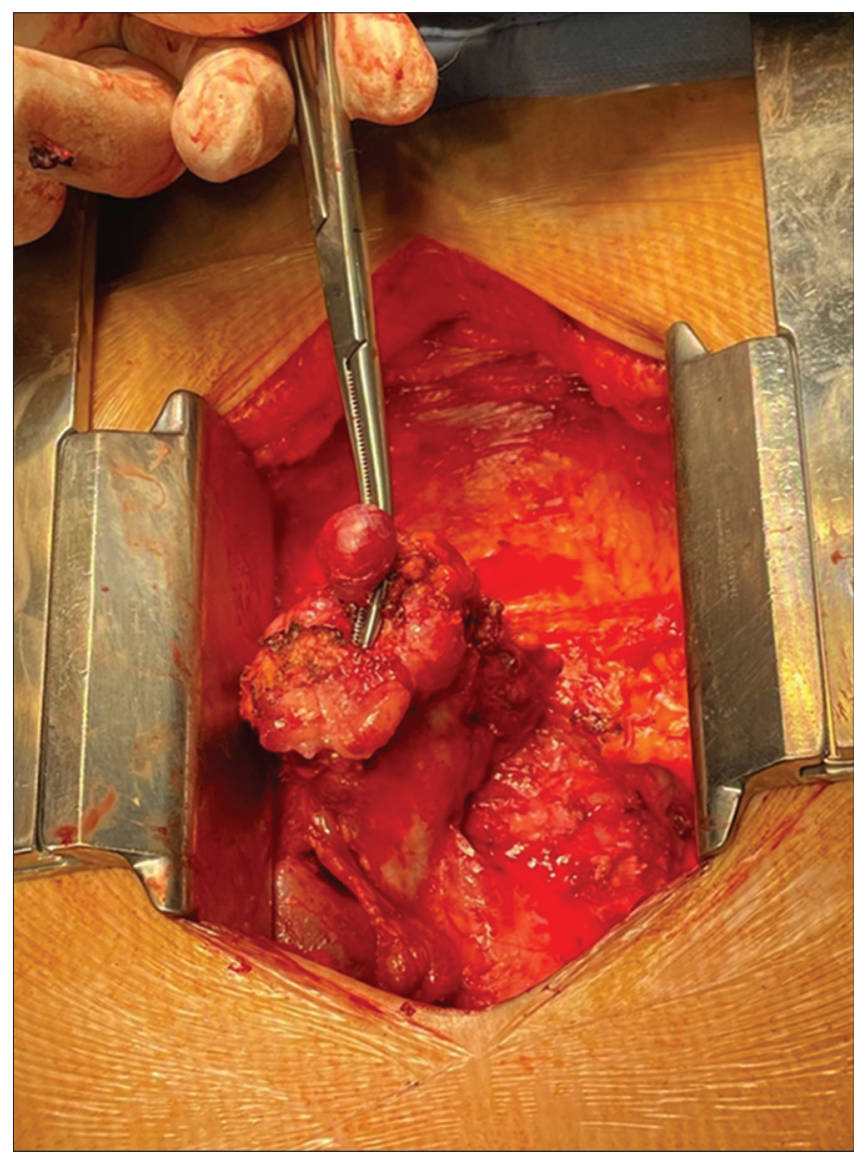

Figure 1: Thymectomy, through the median sternotomy
During treatment, the patient's condition tended to be stable and the patient was discharged after 5 days of treatment and hospital monitoring. No significant abnormalities were found on physical examination. The patient went home under oral therapy with pyridostigmine (Mestinon $60 \mathrm{mg} \times 4$ and corticosteroids in small doses).

\section{Discussion}

Myasthenia gravis is believed to be the most common progressive autoimmune disorder involving the postsynaptic junction [1]. It has a varying degree of involvement ranging from the ocular or other localized regions to a global involvement. Many patients are absent of active symptoms or are in remission throughout their unique course of the disease. With the chronic and progressive destruction of the postsynaptic acetylcholine receptors (AChR), inadequate depolarization occurs at the neuromuscular junction (NMJ) to trigger a muscular action potential, which leads to an overall decrease in neuromuscular transmission. Initially, muscle strength is strongest with voluntary movements but quickly decreases as acetylcholine release tapers. Significant portions of MG cases are associated with an lgG antibody against the AChR [1]. These antibodies reduce the number of functional receptors by several mechanisms. First, cross-linking of receptors with the IgG antibodies increases receptor destruction [1]. Second, the antibodyreceptor complex directly causes damage to the NMJ membrane [1]. Another portion of the MG cases that are negative for IgG antibodies have antibodies against the musclespecific receptor kinase at the NMJ [2]. Destruction of this kinase leads to developmental abnormalities of the NMJ, which results in the functional lack of AChR. The presented case was not tested for antibodies of either type because this patient had longstanding MG.

Even though a definitive trigger of MG remains to be discovered, patients with MG tend to have some abnormality of their thymus gland, which may include thymic hyperplasia or a thymoma [1]. Ptosis is often a predisposing symptom that may stay confined and be self-limiting or convert to a generalized form in the latter course of the disease as in the presented case. Distal limb muscular involvement occurs less frequently than in proximal muscles [2]. Respiratory compromise is rarely present in isolation but may affect some patients and is termed a Myasthenic crisis when control of the airway is necessary (ie, tracheal intubation and mechanical ventilation) [1]. The patient in this case lacked apparent signs and symptoms of active pulmonary compromise at the time of evaluation. 
Based on the age of the patient when symptoms first appeared, MG was classified as earlyonset if the age of onset is below 50 years and lateonset for more or equal to 50 years [4]. Neuromuscular symptoms in early-onset MG tend to be less severe and less associated with thymoma compared to late-onset MG [4], [5].

Plasmapheresis is another mainstay therapy for MG [6], [7]. Research conducted by Nagayasu et al. [7] showed that the rate of post-thymectomy pharmacological remission in the group undergoing plasmapheresis reached $79 \%$ compared to $50 \%$ in the group not undergoing plasmapheresis. Although effective in relieving symptoms, the effects of plasmapheresis are temporary due to the ongoing process of antibody resynthesis [8]. The use of plasmapheresis is also hindered due to possible side effects (hypotension, coagulopathy, etc.) and the high costs [9]. The role of plasmapheresis in improving outcome of thymectomy is still controversial.

Anesthetic point of view: Several proposed techniques avoid the pitfalls of having to manage neuromuscular blocking agents. The use of propofol or sevoflurane with opioids without the use of any neuromuscular blocking agents has been used with success [10]. Both of these approaches allowed for the successful and uneventful extubation of patients in the operating room or ICU at the end of the procedure [10]. The successful and uneventful outcome of the presented case with propofol and sevoflurane further reinforces this technique. Propofol alone for induction and maintenance of anesthesia was adequate in a number of MG cases that also allowed for early extubation and no need for postoperative mechanical ventilation [10]. Perhaps the most important consideration for the patient with $M G$ is determining which anesthetic agents to use that avoid any possible depression in muscular function. The current literature supports a thorough preoperative evaluation to gauge the extent of disease, current treatment, and the involvement of other systems, especially cardiovascular and respiratory systems. The patient in the presented case had a thorough investigation of all systems to evaluate extent of the present disease. Perhaps, the most important are the cardiovascular and pulmonary systems, which proved to be normal in this case. The anesthetic of choice may be the one that avoids neuromuscular blocking agents and uses shortacting induction agents. Perhaps, vigilant peripheral neuromuscular monitoring is of more importance if neuromuscular blocking agents must be used. This is an important note because close monitoring of the NMJ by a peripheral nerve stimulator better allows for reduced dosages to achieve the same degree of muscular relaxation. The presented case avoided the use of neuromuscular blocking agents.

\section{Conclusion}

Generalized myasthenia gravis is a rare disease. The primary management is antiacethylcholinesterase drugs. Early diagnosis and treatment might increase patient's chance for full remission. Thymectomy is a common procedure performed in cases of myasthenia gravis (MG) with a thymoma or general MG that does not improve with medical therapy. This procedure is usually preceded by plasmapheresis to prevent the occurrence of myasthenic crisis and improve clinical outcomes after thymectomy. Early-onset MG has a different phenotype than late-onset MG. Medical treatment for all patients in the form of anticholinesterase drugs and corticosteroids depending on the severity of the disease preoperatively including three sessions of plasmapheresis has shown to be effective before thymectomy.

The use of propofol infusion and endotracheal anesthesia in transsternal thymectomy provides good operative and postoperative conditions, and the use of modified nonmuscle relaxant technique provides excellent intubating, operative, and postoperative conditions. We believe that for such procedure an established protocol which involves perioperative management is essential for better outcome.

Transsternal thymectomy is feasible in the management of patients with MG at all stages with high improvement rate and low surgical morbidity. Those patients aged 35 years or less at operation, with symptoms developed <24 months before operation, may benefit more from thymectomy.

\section{References}

1. Hirsch NP. Neuromuscular junction in health and disease. $\mathrm{Br} \mathrm{J}$ Anaesth. 2007;99(1):132-8.

PMid:17573397

2. Abel M, Eisenkraft JB. Anesthetic implications of myasthenia gravis. Mt Sinai J Med. 2002;69(1-2):31-7. PMid:11832968

3. Hines RL, Marschall KE. Anesthesia and Co-existing Disease. $5^{\text {th }}$ ed. Philadelphia, PA: Churchill Livingstone; 2008.

4. Wang W, Chen YP, Wei DN. The clinical characteristics of earlyonset versus late-onset types of myasthenia gravis. Zhonghua Nei Ke Za Zhi. 2011;50(6):496-8.

PMid:21781535

5. Romi F, Gilhus NE, Aarli JA. Myasthenia gravis: Clinical, immunological, and therapeutic advances. Acta Neurol Scand. 2005;111(2):134-41. https://doi. org/10.1111/j.1600-0404.2005.00374.x

PMid:15644074 
6. Ropper $\mathrm{AH}$, Brown RJ. Adams and Victors Principles of Neurology. $10^{\text {th }}$ ed. New York: McGraw Hill Professional; 2014.

7. Nagayasu T, Yamayoshi T, Matsumoto K, Ide N, Hashizume S, Nomura M, et al. Beneficial effects of plasmapheresis before thymectomy on the outcome in myasthenia gravis. Jpn J Thorac Cardiovasc Surg. 2005;53(1):2-7. https://doi.org/10.1007/ s11748-005-1001-y

PMid:15724495

8. El-Bawab H, Hajjar W, Rafay M, Bamousa A, Khalil A, Al-Kattan K. Plasmapheresis before thymectomy in myasthenia gravis: Routine versus selective protocols. Eur J Cardiothorac Surg.
2009;35(3):392-7. https://doi.org/10.1016/j.ejcts.2008.11.006

PMid: 19136275

9. Mozaffar M, Fard AA, Kharazm P, Kermani HJ, Aminserest M, Yavari P. Thymectomy after plasmaphersis in myasthenia gravis: Results of long term follow up. Tanaffos. 2007;6(1):23-8.

10. Rocca GD, Coccia C, Diana L, Pompei L, Costa MG, Tomaselli E, et al. Propofol or sevoflurane anesthesia without muscle relaxants allow the early extubation of myasthenic patients. Can J Anaesth. 2003;59(6):547-52. https://doi.org/10.1007/ bf03018638

PMid: 12826544 